\title{
Research on the Reliability of Evaluating the Efficiency of Commanding Information System
}

\author{
Liu Jing-Xue, Li Hong \\ PLA Academy of National Defence Information, Wuhan,China. \\ E-Mail: 1jx_62@sohu.com
}

\begin{abstract}
When evaluating the efficiency of the commanding information system, the results are always questioned because of the fuluctuation of the data used. The article introduced a method which is suitable to measure the reliability of the evaluation efficiency of the commanding information system as per its own characteristic. Finally, according to the calculation of a sample, it is proved that this mehod of measuring the reliability of the evaluation efficiency performs very well and it is practible and workable in reality.
\end{abstract}

Keywords-Commanding information system; Evaluating efficiency; Measuring reliability.

\section{INTRODUCTION}

The new joint battle mainly based on information warfare, as the command and control platform, the Commanding Information System (Hereafter called "CIS") becomes more and more important in armies of all countries. The main objective of CIS is to enhance the overall warfare efficiency of all units by improving their capabilities of commanding battle, control of weapons, reconnaissance and exploration, rapid response, and apperceiving situation. Evaluating the efficiency of CIS is an important topic to determine whether or not it could meet the information commanding and controlling demand in warfare. Through scientifically and reasonably evaluating the efficiency of CIS, the commanders can easily figure out the weakness in CIS so as to improve the performance of CIS in wartime or peacetime, which is helpful to improve their commanding abilities, and it has significant influence of the development of CIS and improve the overall efficiency. Today, there are a lot of articles introduced evaluation of the efficiencies of various information systems, but most of them did not consider the fluctuation of data in evaluation ${ }^{[1-4]}$, only few articles touched the topic of the reliabilities of the evaluation efficiency of information systems. In general, the efficiency of CIS is measured by various performance indexes comprehensively. So, this article first introduces a method to evaluate the efficiency of CIS from the view of synthetic evaluation Then, the reliability of the evaluation efficiency of CIS is studied through simulative calculation. The method introduced in this article considers the fluctuation of data in evaluating CIS, which is more reliable and could determine the evaluation efficiency more objectively and reliably.

\section{THE METHOD OF EVALUATING THE EFFICIENCY OF CIS}

\section{A. The Index Structure}

According to the demand of modern warfare and the principles of generality, availability and stability, the index architectonic structure of evaluating the efficiency of CIS can be established as shown as table $1^{[4]}$.

TABLE 1. THE INDEX ARCHITECTONIC STRUCTURE OF EVALUATING THE EFFICIENCY OF THE CIS

\begin{tabular}{|c|c|}
\hline $\begin{array}{l}\text { The first } \\
\text { level index }\end{array}$ & The second level index \\
\hline $\begin{array}{l}U_{1} \text { - the capability } \\
\text { of command and } \\
\text { control }\end{array}$ & $\begin{array}{l}U_{11} \text { - the command delay time; } U_{12}- \\
\text { the quality of command personnel; } \\
U_{13} \text { - the scope of control battle; } U_{14} \\
\text { the skill of assistant decision-making. }\end{array}$ \\
\hline $\begin{array}{l}U_{2} \text { - the capability } \\
\text { of communications }\end{array}$ & $\begin{array}{l}U_{21} \text { - the signal covering scope; } U_{22}- \\
\text { the communications capacity; } U_{23} \text { the } \\
\text { integrative intercommunication; } U_{24} \\
\text { the reliability. }\end{array}$ \\
\hline $\begin{array}{l}U_{3} \text { - the capability } \\
\text { of reconnaissance } \\
\text { and exploration }\end{array}$ & $\begin{array}{l}U_{31} \text { - the scope of reconnaissance and } \\
\text { exploration; } U_{32} \text { the advanced } \\
\text { technique means; } U_{33} \text {-the probability } \\
\text { of detecting objective; } U_{34} \text { the } \\
\text { probability of void alert. }\end{array}$ \\
\hline $\begin{array}{c}U_{4} \text { the mobile } \\
\text { capability }\end{array}$ & $\begin{array}{l}U_{41} \text { - the movement ability; } U_{42} \text { the } \\
\text { competence of rapid regrouping; } U_{43}- \\
\text { the orientation competence. }\end{array}$ \\
\hline $\begin{array}{l}U_{5} \text { the capability } \\
\text { of information } \\
\text { support }\end{array}$ & $\begin{array}{l}U_{51} \text { - the ratio of sharing resources; } \\
U_{52} \text { the competence of acquiring } \\
\text { information; } U_{53} \text { the speed of } \\
\text { renovating information. }\end{array}$ \\
\hline
\end{tabular}

\section{B. The Evaluating Model}

When the efficiency of CIS is evaluated, the comment set of fuzzy language should be first determined, and the benchmark values of all second level indexes that aim at each comment are subsequently determined. Then, the performances of CIS are tested, and some experts utilize the gained data to give the scores of all second level indexes according to the grading standard shown in table 2. Finally, the elementary evaluation result is made upon the basis of 
calculating the weighted difference value between the scores given by the experts and the benchmark values.

\section{TABLE 2. THE GRADING STANDARD}

\begin{tabular}{|c|c|}
\hline Judgment value & Graded interval \\
\hline extreme good & {$[90,100]$} \\
\hline very good & {$[80,90)$} \\
\hline good & {$[70,80)$} \\
\hline comparatively good & {$[60,70)$} \\
\hline moderate & {$[40,60)$} \\
\hline comparatively poor & {$[30,40)$} \\
\hline poor & {$[20,30)$} \\
\hline very poor & {$[10,20)$} \\
\hline extreme poor & {$[0,10)$} \\
\hline
\end{tabular}

Given that $w_{i}$ is the weight of $U_{i}, w_{i}$ should be determined by the commander $(i=1,2, \ldots, 5)$.Let $V=\left\{v_{1}, v_{2}, \ldots, v_{L}\right\}$ stand for the comment set determined in advance, the comments in $V$ rank decreasingly, namely $v_{1} \succ v_{2} \succ \ldots \succ v_{L}$. Aiming at $v_{k} \in V$, the determined benchmark value of $U_{i j}$ is denoted as $b_{i j}^{k}(k=1,2, \ldots, L$; $\left.i=1,2, \ldots, 5 ; j=1,2, \ldots, i_{k}\right)$. Given that $e_{i j}(i=1,2, \ldots, 5$; $\left.j=1,2, \ldots, i_{k}\right)$ is the score of $U_{i j}$, which is determined by the evaluation experts according to the situation of testing the CIS. If $q \in\{1, \ldots, L\}$ meets

$$
\sum_{i=1}^{5} \sum_{j=1}^{i_{k}} w_{i}\left(e_{i j}-b_{i j}^{q-1}\right)<0 \leq \sum_{i=1}^{5} \sum_{j=1}^{i_{k}} w_{i}\left(e_{i j}-b_{i j}^{q}\right)
$$

then the efficiency of CIS achieves the standard of $v_{q}$ as a whole, $v_{q}$ is accepted to be the elementary evaluation result.

\section{THE RELIABILITY OF EVALUATING THE EFFICIENCY OF CIS}

Because there are possibly some warps in the tested data and the experts' judgments, It is hard to avoid definite fluctuation in determining the score of each second level index. Consequently, It is necessary that the commander organize other experts to measure the reliability of $v_{q}$. So, $e_{i j}$ should be surveyed by these experts $(i=1,2, \ldots, 5$; $\left.j=1,2, \ldots, i_{k}\right)$, and $e_{i j}$ is classified according to the standard included in \{"lesser warp", " obvious warp", "biggish warp"' .

\section{A. Measuring the Reliability of $v_{q}$}

In order to measure the reliability of $v_{q}$, it is demanded to make a good deal of simulative tests. As shown in [5], it is resultful to rely on the even design, in which a even distributing point $e_{i j}^{\prime}$ that is nearby $e_{i j}$ is used to substitute for $e_{i j}$. If $e_{i j}$ is considered to be precise by the experts, then let $e_{i j}^{\prime}=e_{i j}$. According to table 2 , if $e_{i j}$ is considered to be lesser warp, let $e_{i j}^{\prime}=e_{i j}-1+2 \times \xi_{i j}$ when $e_{i j}$ is considered to be fluctuant, let $e_{i j}^{\prime}=e_{i j}+\xi_{i j}$ when $e_{i j}$ is considered to be up floating, let $e_{i j}^{\prime}=e_{i j}-\xi_{i j}$ when $e_{i j}$ is considered to be down floating, where $\xi_{i j}$ is a random number obeying 0-1 uniformity distribution. Analogously, if $e_{i j}$ is considered to be obvious warp, let $e_{i j}^{\prime}=e_{i j}-2+4 \times \xi_{i j}$ when $e_{i j}$ is considered to be fluctuant, let $e_{i j}^{\prime}=e_{i j}+2 \times \xi_{i j}$ when $e_{i j}$ is considered to be up floating, let $e_{i j}^{\prime}=e_{i j}-2 \times \xi_{i j}$ when $e_{i j}$ is considered to be down floating. Similarly, if $e_{i j}$ is considered to be biggish warp, let $e_{i j}^{\prime}=e_{i j}-3+6 \times \xi_{i j}$ when $e_{i j}$ is considered to be fluctuant, let $e_{i j}^{\prime}=e_{i j}+3 \times \xi_{i j}$ when $e_{i j}$ is considered to be up floating, let $e_{i j}^{\prime}=e_{i j}-3 \times \xi_{i j}$ when $e_{i j}$ is considered to be down floating. Making a simulative calculation by substituting $e_{i j}^{\prime}$ for $e_{i j}$ in the both sides of formula (1), if formula (1) comes into existence, then this simulative calculation supports $v_{q}$, otherwise, it does not support $v_{q}$. Suppose each round contains $N$ simulative calculations and $M$ rounds have been made. Given that $N_{i}$ denotes the times of simulative calculations supporting $v_{q}$ in ith round. As a result, the reliability of $v_{q}$ in ith round can be defined as follows:

$$
C_{i}=\frac{N_{i}}{N} \quad(i=1,2, \ldots, M)
$$

Therefor, the reliability of $v_{q}$ derived from the simulative calculations in $M$ rounds can be considered as follows:

$$
C^{q}=\min \left\{C_{1}, C_{2}, \ldots, C_{M}\right\}
$$

\section{B. A Calculative Example}

Given that $V=\{$ "very good", "good", "Comparatively good", "Moderate", "Comparatively poor", "poor", "Very poor". $\}$ is the comment set determined in advance for evaluating the efficiency of one CIS, the weight vector of the first level index determined by the commander is $W=(0.2821,0.0753,0.1133,0.2325,0.2968)$, the benchmark value of each second level index that corresponds to each comment in $V$ is the middle value of the corresponding graded interval shown in table 2. Suppose that the scores of all second level index given by the 
evaluating experts are : $e_{11}=82, e_{12}=75, e_{13}=85$, $e_{14}=90, e_{21}=75, e_{22}=85, e_{23}=90, e_{24}=80$, $e_{31}=75, e_{32}=70, e_{33}=75, e_{34}=85, e_{41}=78$, $e_{42}=72, e_{43}=75, e_{51}=78, e_{52}=75, e_{53}=80$. It is required to evaluate the efficiency of the CIS. Assumed that after having reviewed the scores of all second level index, the inspecting experts consider: $e_{21}$ and $e_{23}$ have biggish warps, the score of $e_{23}$ is on the high side, the score of $e_{21}$ is on the low side. $e_{11}, e_{32}$ and $e_{41}$ have obvious warps, the score of $e_{32}$ is on the low side, the scores of $e_{11}$ and $e_{41}$ are fluctuant. $e_{24}$ and $e_{53}$ have lesser warps, their scores are fluctuant. The other scores are comparatively precise. Let $N=100000$ and $M=10$, It is required to determine the reliability of this evaluation.

Looking at $v_{1}=$ "Very good" and $v_{2}=$ "good", the benchmark values of $U_{i j}$ derived from the graded interval in table 2 are respectively $b_{i j}^{1}=85$ and $b_{i j}^{2}=75(i=1,2, \ldots, 5$; $\left.j=1,2, \ldots, i_{k}\right) . \because \sum_{i=1}^{5} \sum_{j=1}^{i_{k}} w_{i}\left(e_{i j}-b_{i j}^{1}\right) \approx-20.48<0$, but $\sum_{i=1}^{5} \sum_{j=1}^{i_{k}} w_{i}\left(e_{i j}-b_{i j}^{2}\right) \approx 14.23>0, \therefore$ From formula (1), the efficiency of the CIS achieves the standard of $v_{2}=$ "good" as a whole, and the elementary evaluation result is "good".

According to the judgments of the inspecting experts, it is required to generate seven random numbers obeying $0-1$ uniformity distribution, namely $\xi_{14}, \xi_{23}, \xi_{11}, \xi_{41}$, $\xi_{21}, \xi_{24}, \xi_{53}$. Let $e_{21}^{\prime}==e_{21}+3 \times \xi_{21}, e_{23}^{\prime}=e_{23}-$ $3 \times \xi_{23}, \quad e_{32}^{\prime}=e_{32}+2 \times \xi_{32}, \quad e_{11}^{\prime}=e_{11}-2+4 \times \xi_{11}$, $e_{41}^{\prime}=e_{41}-2+4 \times \xi_{41}, e_{24}^{\prime}=e_{24}-1+2 \times \xi_{24}, e_{53}^{\prime}=e_{53}$ $-1+2 \times \xi_{53}$, the rest $e_{i j}^{\prime}=e_{i j}$. Calculating $K=$ $\sum_{i=1}^{5} \sum_{j=1}^{i_{k}} w_{i}\left(e_{i j}^{\prime}-b_{i j}^{2}\right)$, if $K \geq 0$, then the result of this simulative calculation supports $v_{2}$. By programming to calculate ten rounds that each round includes a million simulative calculations, the reliabilities of this ten rounds are in turn $0.940,0.963,0.922,0.903,0.873,0.813,0.863$, $0.858,0.943,0.977$.

\section{The Confirmation and Revision ofthe Evaluation Result}

To measure the reliability of $v_{q}$ is helpful for confirmation and revision of $v_{q}$. Suppose $\alpha$ is a threshold given by the commander ( $\alpha \geq 0.6$ ), if the reliability of $v_{q}$ satisfies:

$$
C^{q} \geq \alpha
$$

then $v_{q}$ should be confirmed, otherwise the evaluation result should be degraded, namely the evaluation result should be revised as $V_{q+1}$, the reliability of $v_{q+1}$ needs to be measured again, and $v_{q+1}$ needs to be reconfirmed.

\section{CONCLUSION}

According to the characteristics of CIS, this article briefly analyzed the index architectonic structure that evaluates the efficiency of CIS, established the evaluation model from the view of general multi-performance indexes, and then conducted a method of measuring the reliability of evaluating the efficiency. The calculative example shows that the methods introduced in this article can measure the reliability of the evaluation efficiency of CIS easily by combining the operation of CIS and the experts' judgments. Through simulative calculations, the reliability of the evaluation result can be figured out effectively and its flexibility and practicality has been proved. It is worthy of explaining that the evaluation result can be easily revised and confirmed according to the reliability, which is helpful for enhancing the accuracy of this evaluation result and discovering possible hidden problems so as to improve CIS. Moreover, although the methods in this article are to aim at CIS, they can be introduced to common information systems to improve the objectivity of evaluating the efficiency of information systems.

\section{ACKNOWLEDGMENT}

This article is supported by the PLA Academy of National Defence Information, the Computer Science and Electronic Technology International Society and the IEEECPS.

\section{REFERENCES}

[1] Hu Yu-nong, Xia Zeng-hong, Wang Jun-feng,etc. Methods summary for performance evaluation of complex electronic information system[J]. Application Research of Computers. 2009,26(3): 819 822.

[2] Li Zhi-meng, Tan Qun, Wang Yan-ming, etc. Effectiveness Evaluation Method for Information Systems Based on Exploratory Analysis[J]. Science Technology and Engineering. 2009,9(22): 6702 6706.

[3] Chu Jian-feng, Zhang Yao-hong, Chen Liang. Effectiveness Evaluation of Artillery Command Information System Based on Fuzzy AHP Method[J]. Ship Electronic Engineering. 2010,30 (6) : 41 44.

[4] Liu Jing-xue. Dynamic Evaluating Research on the Efficiency of Commanding Information System [C].2012 International Conference on Intelligent Systems Design and Engineering Applications. 6-7 January 2012, pp:813 $\sim 816$.

[5] Fang Kai-tai, Wang Yuan. Theory and Applications of "Even Distributing Point"[A]. Qiu Chen-tong, Yang Le, Ji Li-zhen. Mathematics exist in anywhere[C]. Beijing: Higher Education Press. 2012, pp: $66 \sim 72$. 